\title{
Home Monitoring of Cardiac Devices in the Era of COVID-19
}

\author{
Jennifer C. Miller ${ }^{1} \cdot$ Devin Skoll $^{1} \cdot$ Leslie A. Saxon $^{1}$ (D) \\ Accepted: 11 November 2020 / Published online: 20 November 2020 \\ (C) Springer Science+Business Media, LLC, part of Springer Nature 2020
}

\begin{abstract}
Purpose of Review Despite the promise of remote patient monitoring (RPM), this technology remained underutilized secondary to a lack of data transparency and systems issues until the COVID-19 pandemic ushered in a new era of telehealth and virtual solutions out of necessity. This review will explore the data supporting the use of RPM via both implantable and wearable devices in the field of cardiology and the role of home monitoring using RPM in the era of COVID-19.

Recent Findings RPM using implantable cardiac devices is a safe alternative to in-person only visits which leads to enhanced patient satisfaction and improved clinical outcomes. Consumer-grade wearable sensors have drastically expanded RPM capabilities from just the sickest cardiac patients to the entire population aiding in early diagnosis and real-time disease management. Summary Home monitoring enabled by automated alert systems tailored specifically to the needs of the patient by the provider will be the cornerstone of a more continuous, patent-centric healthcare model.
\end{abstract}

Keywords Remote patient monitoring - Implantable cardiovascular sensors and devices · COVID-19 $\cdot$ Hemodynamic monitoring $\cdot$ Heart failure $\cdot$ Arrhythmia

\section{Introduction}

A new era of cardiac management began in 1960 with the creation of the first implantable pacemaker which has since saved countless lives and transformed the practice of medicine [1]. This life-saving technology evolved over the next 60 years to include implantable cardiac defibrillators (ICDs) with capabilities such as cardiac resynchronization therapy (CRT) enabling medical providers to safely manage patients with dangerous arrhythmias and congestive heart failure outside the confines of the hospital. A pivotal step in the advancement of this technology was the implementation of remote patient monitoring (RPM). One of the first and the largest RPM studies, the

This article is part of the Topical Collection on Invasive Electrophysiology and Pacing

Leslie A. Saxon

saxon@usc.edu

Jennifer C. Miller

jenmiller@chla.usc.edu

Devin Skoll

dskoll@usc.edu

1 University of Southern California's Center for Body Computing, 12015 E Waterfront Dr, Los Angeles, CA 90094, USA
ALTITUDE study, demonstrated a 50\% reduction in 1- and 5year mortality rates for patients whose devices were followed remotely versus those with a standard in-person follow-up [2]. Subsequent studies confirmed this survival benefit [3] and demonstrated survival could be further increased with better patient compliance (completion of $>75 \%$ of weekly transmissions) [4]. Today, RPM has become the standard of care for patients with implantable cardiac devices [5] and the field has rapidly expanded to include implantable sensors, such as pulmonary artery pressure monitors and implantable loop recorders, as well as wearable monitors and external smart-phone enabled devices. These technologies along with cloud computing tools permit continuous virtual monitoring of all patients breaking healthcare free from its traditional brick and mortar model and facilitating a holistic and comprehensive "lifecare" model [6]. Prior to 2020, the lifecare model remained unrealized secondary to outdated and restrictive regulations at the local, state, and federal level as well as limited reimbursement strategies; however, the emergence of the COVID-19 virus and the first truly global pandemic has led to the removal of these barriers [7] and forced the healthcare system to transform and innovate at an unprecedented speed.

Cardiac patients, particularly those with heart failure and implantable devices, are at increased risk for COVID-19 morbidity and mortality $[8 \bullet \bullet, 9 \bullet]$. There has been a notable decrease in admissions for heart failure and heart attacks which 
may be secondary to patient reluctance to visit healthcare settings. This may paradoxically result in an increased number of high acuity admissions in the future [10]. Furthermore, there is growing evidence to suggest COVD-19 has significant and even long-term cardiac effects which may lead to an echo pandemic of heart failure patients [11]. Given the field's long history of utilizing implanted, wearable, and external technologies for RPM, cardiology has been uniquely well equipped to transition traditional healthcare visits to virtual telehealth visits and take advantage of digital health tools. This article will review data on clinical outcomes associated with remote monitoring of cardiac implantable electronic devices (CIED) and implantable sensors, such as the pulmonary artery pressure sensors and implantable loop recorders (ILRs), as well as the feasibility of utilizing wearable sensors and external devices for remote cardiac monitoring. It will review the known and suspected cardiac clinical effects of COVID-19 virus. Finally, it will reevaluate the role of virtual solutions and home monitoring for cardiac patients in this new, rapidly disrupting healthcare model.

\section{Remote Monitoring in Patients with CIEDs}

Remote patient monitoring continuously surveys millions of CIED patients connecting them with their care teams and facilitating a rapid response for urgent clinical and device technical issues thus leading to improved outcomes and cost benefits [6]. RPM has been endorsed as a safe alternative to in-person only visits in consensus statements across the world $[5,12,13 \bullet \bullet, 14]$. There are high levels of patient satisfaction with RPM, and there is evidence to suggest RPM may enhance patient and clinical reported outcomes [15-17]. RPM was first introduced as a safe means to detect pacemaker system malfunctions [18, 19], but it soon became apparent this technology had the ability to reduce times to a clinically actionable event $[20,21]$ with possible profound implications on patient outcomes. RPM via the ALTITUDE registry and Merlin network databases amassed massive amounts of patient data, permitting large scale analytics on scales not seen before to demonstrate decreased mortality in remotely monitored patients with pacemakers, ICDs, and CRT. These data have been a rich resource to develop novel strategies for disease management and device features [22, 23].

CIEDs incorporate features beyond rhythm sensing and self-diagnostics including activity level, thoracic impedance, and other single or combined surrogate markers for pulmonary congestion (i.e., HeartLogic ${ }^{\mathrm{TM}}$, Boston Scientific, Marlborough, MA). The randomized controlled trial INTIME demonstrated a significant survival improvement in heart failure patients with CIEDs with automatic, daily, implant-based RPM likely secondary to earlier arrhythmia detection as well as heart failure decompensation detection, recognition of suboptimal device function, and increased patient contact with their healthcare team via telemonitoring alerts [16]. Conversely, the REM-HF study of heart failure patients with CIEDs from the three major manufactures showed no difference in mortality or hospitalization but notably performed weekly instead of daily transmissions [24]. The COMMIT-HF study evaluated similar devices, but with predominantly daily transmissions, and demonstrated a significant reduction in one-year mortality over 3 years of follow-up [25]. A meta-analysis of RPM trials demonstrated that RPM was at least non-inferior to in-person visits, and noted that earlier detection of clinical events and decreased mortality were likely dependent on the frequency of RPM and an established response mechanism to RPM generated alerts [26]. The real promise is in combining electrical measures with validated hemodynamics measures to more completely understand the status of a CIED patient from an electrical and hemodynamic perspective. This is particularly relevant for patients with primary prevention ICD's and CRT devices, who have reduced ejection fraction and heart failure.

In addition to the intended benefit of identification and treatment of ventricular arrhythmias, CIEDs have the added capability of identifying supraventricular tachyarrhythmias such as atrial fibrillation leading to earlier identification and initiation of therapy $[18,21,27,28]$. This early identification of atrial arrhythmias may have contributed in part to a reduction seen in hospitalizations for the management of atrial arrhythmias and strokes in the COMPAS trial [18]. Furthermore, thromboembolic events in a large CIED population followed for 4 years were found to have less than half the incidence of thrombotic events predicted based on their CHA2DS2-VASc score [29]. Conversely, the IMPACT trial found no difference in the prevention of thromboembolism or bleeding with early initiation and interruption of anticoagulation in patients with CIEDs and remotely detected atrial tachyarrhythmias [30]. This study highlights the inconvenient truth that current treatment guidelines are based on data from predominately symptomatic patients collected over finite periods of time and, in the current paradigm shift to continuous, remote monitoring of data, a reevaluation of what is considered "sick" and "healthy" may lead to a dramatic change in the way patients are medically managed.

\section{Hemodynamic Monitoring in Patients with Implantable Sensors}

One of the earliest and most sensitive measures of worsening heart failure is increased intracardiac pressure secondary to volume overload, and early identification of elevated intracardiac pressures is believed to essential in the establishment of timely intervention leading to improved patient outcomes [31]. These measures worsen well in advance of symptoms development, and therefore represent a powerful tool to 
prevent heart failure decompensation and hospitalization. To date, the CardioMEMS (Abbott Labs) is the only FDAapproved wireless implantable pulmonary artery pressure (PAP) sensor for management of symptomatic heart failure [32]. Providers can remotely monitor their patient's hemodynamic status and provide timely, personally tailored treatments that are associated with better symptoms management and improved event-driven outcomes [33-35, 36•]. Remote PAP monitoring augments ambulatory treatment of patients with heart failure, decreasing hospitalizations, and overall costs of heart failure management [37, 38]. Despite the connected capabilities of this device and CIEDs, patients do not have access to these data and must rely on the clinic and healthcare team to follow and evaluate any changes inhibiting the potential real-time capabilities of such technology. The LAPTOP-HF study evaluating the role of an implantable left atrial pressure sensor was the first major study to fully leverage real-time patient implantable sensor data. It provided patients direct access to their pressure information in addition to automated, dynamic medical recommendations based on individually tailored programs developed by heart failure specialists. The trial was stopped after enrolling nearly 500 patients due to perceived safety concerns by the Data Safety and Monitoring Board; however, at a mean of 2 years follow-up, patients had significantly reduced heart failure hospitalizations, an effect comparable or superior to results obtained in clinical trials with PAP sensors. Furthermore, procedural complications were later evaluated to be below the safety threshold [6, 34-36]. The recently published largest PAP study, the CardioMEMS Post-Approval study, similarly found a low rate of the procedure and device-related complications. The study demonstrated a decline in PAP, 57\% reduction in heart failure hospitalizations, and $26 \%$ reduction in all-cause hospitalization in the year post-implantation regardless of sex, race, and starting ejection fraction. Importantly, there was excellent patient compliance with a pressure transmission rate of $85 \%$ daily and $100 \%$ weekly [39•]. Providing patients with the tools to continuously monitor and manage their medical condition, under the guidance of their healthcare team, empowers them to be active participants in their own care allowing for better disease control and improved clinical outcomes.

\section{Wearable ECG Sensors and Studies for Atrial Fibrillation}

CIEDs and implantable loop recorders (ILRs) have demonstrated the utility of continuous RPM to identify asymptomatic or mildly symptomatic atrial fibrillation but are significantly limited by their invasive nature for use in the general population [40]. Homebased ECG monitoring in individuals at risk for atrial fibrillation (AF) using the wearable sensor Zio Patch (iRhythm Technologies) first demonstrated the important role of home monitoring using wearable sensors in earlier AF diagnosis and initiation of anticoagulants. Unfortunately, the ZioPatch is only continuous in its data collection for up to 2 weeks and does not have real-time connectivity [41, 42]. Other consumer enabled devices such as the FDA- and CEapproved AliveCor Kardia devices deploy an artificial intelligence-driven rate and rhythm detection algorithm to identify new cases of AF at an almost 4-fold increase in detection rate compared to routine care in at-risk populations [43-47]. These devices allow consumers to assess their rhythm on-demand though notably does not provide continuous rhythm monitoring. With the FDA clearance of algorithmbased detection of AF on the Apple Watch Series 4, a tool for arrhythmia detection was placed directly into the hands of the consumer, who for the first time had the ability to passively collect truly continuous heart rate data and serve as the gatekeeper to their own health data [48]. The Apple Heart Study was a large siteless study that enrolled 419,000 patients in a mere 8 months and demonstrated $34 \%$ of patients had AF confirmed on a subsequent ECG patch with 0.84 positive predictive value in identifying AF concurrently on the ePatch [49•]. Critics noted the low percentage of patients later confirmed to have AF; however, paroxysmal AF is intermittent in nature and a week-long patch may not measure the heart rhythm long enough to capture an episode. This again calls to question whether the current diagnostic standards and treatments are sufficient comparisons for longitudinal, continuously collected data. The Apple Watch has since gained approval for single-lead ECG capabilities making it possible to immediately assess an irregular rhythm alert on one device [50]. Additionally, Apple has developed three software kits to facilitate medical research and health tracking: HealthKit, ResearchKit, and CareKit. These are essential in creating an ecosystem that permits the patient to safely and privately access their own personal health information as well as related education and communicate seamlessly with their care provider team who can remotely monitor and curate the patient's health plan [51]. Digital platforms and connected wearable sensors create new holistic models of cardiac management centered around the patient and not the hospital system. It remains to be seen whether this technology can accelerate AF diagnosis and improve patient outcomes. The HEARTLINE study, a collaboration between Johnson \& Johnson, Apple, and various clinical experts, is a randomized controlled siteless digital platform study utilizing the Apple Watch and its AF detection algorithm. The study will enroll an at-risk elderly population for AF, to determine if early diagnosis and education related to AF will drive improved clinical outcomes, including stroke [52]. The Heartline study is an example of leveraging both the patient and the widely available consumer devices. This provides an opportunity to identify and track both symptomatic and asymptomatic AF patients and engage them directly in their diagnosis and care using software, potentially leading to earlier initiation 
of anticoagulation therapy, greater adherence, and decreased adverse outcomes.

\section{COVID-19 Pandemic, Accelerating the Digital Disruption of the Practice of Medicine}

Patient-centric platforms enabled by consumer-facing medical-grade sensors, validated alert algorithms, and on-demand educational tools have the potential to create a more continuous and holistic care model. Furthermore, leveraging patients in their diagnosis and care free the healthcare team to practice at the top of their licenses. Since the onset of the COVID-19 pandemic, the USA and the world have suspended non-urgent scheduled visits and hospitalizations and made a transition to virtual health platforms, utilizing wearable sensors at an unprecedented speed with the intention of reducing virus exposure and use of limited supplies of personal protective equipment. Adoption of telehealth has risen steeply from $11 \%$ in 2019 to $46 \%$ currently in the USA alone. Patients will not go back to the in-person only healthcare model of the past with one survey reporting $76 \%$ of consumers to be moderately or highly likely to use telehealth going forward. Providers have also become more comfortable with the telehealth model. They now conduct 50 to 175 times the number of telehealth visits compared to prior to the pandemic with two-thirds reporting more comfort with virtual visits and $57 \%$ noting telehealth more favorably [53]. The recent relaxation in long-standing national and state restrictive laws and regulations, as well as government stimulus packages that have increased reimbursements for remote care, have allowed for virtual care across state lines and rapidly accelerated digital disruption to the healthcare field [7]. Guidance statements issued by experts in electrophysiology and heart failure recommend that every effort should be made to convert visits to telehealth, and further encourage leveraging electronic medical record data as well as data collected via RPM from implantable and wearable devices to remotely manage patients $[8,9]$. This has led to the rapid growth of virtual care platforms created by both the private and public sector, permanently altering the future of cardiac management.

\section{The Direct and Indirect Cardiac Effects of the COVID-19 Virus}

COVID-19 positive patients with chronic cardiac conditions such as heart failure are at an increased risk of morbidity and mortality. It has become increasingly evident that, even in non-cardiac patients, cardiac injury and, particularly in critically ill patients, cardiac brady- and tachyarrhythmias are of particular concern. Two of the initial case reports from Wuhan, China, demonstrated a $20-27.8 \%$ incidence of cardiac injury in hospitalized COVID-19 patients with significantly increased mortality in patients with cardiac injury compared to those without $[54,55]$. Arrhythmias were reported in $16.7 \%$ of patients in Wuhan [56] and $7.9 \%$ of patients in New York City [57]. Critically ill patients were more likely to experience arrhythmia at a strikingly high incidence of $44 \%$ [56]. The exact cause of the increase of cardiac injury and arrhythmias is unclear, but is currently believed to be most likely secondary to systemic effects and possibly from drug side effects [58, 59]. There is growing evidence to suggest COVID-19 may directly affect the heart as well. Atrial and ventricular arrhythmias have been associated with fulminant myocarditis and cardiogenic shock in COVID-19 patients [59-61]. A recent study of 100 recovering patients $\sim 70$ days post COVID-19 diagnosis demonstrated that $78 \%$ of patients had cardiac involvement on MRI of which $60 \%$ had signs of ongoing inflammation [62•]. More data is required to better understand the exact of pathophysiology of COVID-19 and better characterize long-term affects on the heart. However, the possibility of long-term cardiovascular consequences in COVID-19 recovered patients coupled with the reality that non-COVID-19 cardiac patients may be delaying care during the pandemic [37] is concerning for a major future surge of patients with heart failure and other chronic cardiovascular conditions [11]. In the healthcare field's paradigm shift to keep patients out of the hospital to preserve resources and diminish COVID-19 exposure, it is imperative that both known cardiac patients and COVID-19 patients at risk for cardiac complications are provided safe and effective RPM tools.

\section{RPM in the Age of COVID-19}

RPM via CIEDs and implantable devices has long been feasible but limitations including lack of data transparency and systemic issues, particularly the lack of adoption of userfriendly algorithm-driven alert-based systems and absence of protocols on the safe implementation of RPM geared towards home-based care, have led to the under-utilization of this technology $[8,9]$. As these devices become bluetooth enabled, patients should be provided direct access to their personal data as well as tailored education via their mobile device. Available RPM technologies including implantable devices and consumer-facing wearables should be incorporated into the patient care model to aid in arrhythmia detection as well as monitoring for worsening heart failure in the outpatient setting. There has been a push to produce safe, validated RPM tools for COVID-19 monitoring in the outpatient setting. The KardiaMobile-6L (AliveCor, Inc.) recently received FDA emergency clearance for QTc monitoring and was demonstrated as a feasible alternative to ECG QTc measurements in COVID-19 patients treated with hydroxychloroquine, 
lopinavir-ritonavir, and/or azithromycin [63], medications under investigation for treatment of COVID-19 at the time with known increased risk of QT prolongation particularly in combination $[64,65]$. The FDA has further provided emergency approval for multiple remote patient monitors to further enable the collection of physiologic data in this age of social distancing yet there remain major gaps in realizing a virtual healthcare model [66].

New technologies and digital platforms to aid in remote care should be developed and further research on the role of telehealth, continuous data collecting, advanced automotive features, and RPM is needed to guide best practices. Multiple large institutions have developed remote monitoring platforms to monitor COVID-19 patients at home but rely mostly on patient reporting of vital signs and do not provide tools for the continuous monitoring of health data [67-69]. Wearable sensors such as the FitBit and Apple Watch analyze changes in activity and physiological data to predict influenzalike illness in real time with the possibility of aiding in geographic disease surveillance [70, 71•]. Fitbit enrolled over 100,000 patients into a study to determine whether they could identify early signs of COVID-19 infection using an automated algorithm currently under development and 90 days into the study found preliminarily that RPM via the Fitbit can identify almost half of the COVID-19 positive patients at least 1 day earlier than patients become symptomatic [72]. These and other monitors, including the Oura ring (Oura Health Oy) and WHOOP (Whoop, Inc), have been touted as tools to earlier identify signs of COVID-19 infection and decompensation. They have been incorporated in return to play policies of

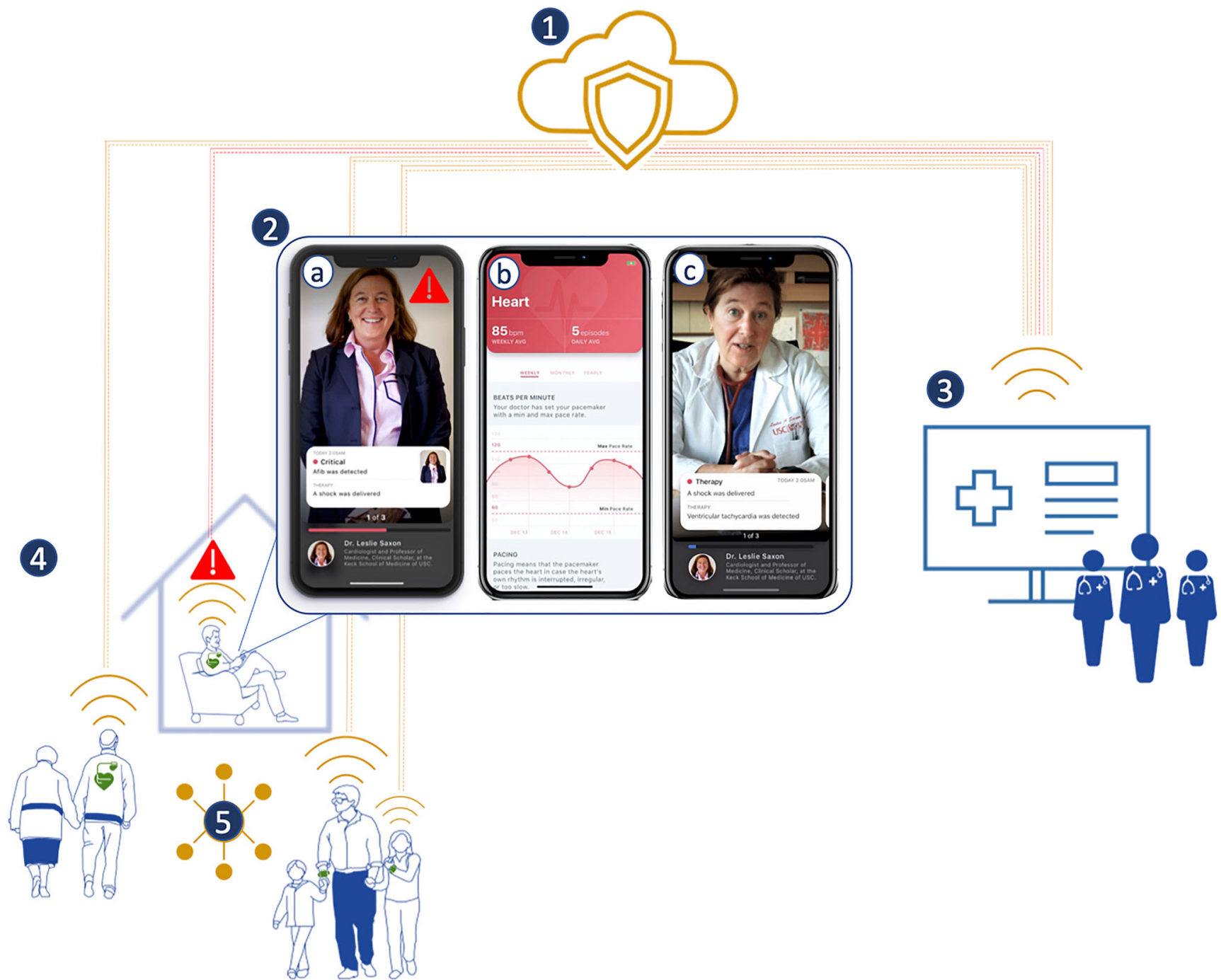

Fig. 1 Remote patient monitoring. Description: 1. Cloud-based remote patient monitoring. 2. Patient-centric secure mobile-based applications. a Real-time algorithm (AI) driven automated alerts to providers and patients. $b$ Medical and cardiac data visualization promoting positive biofeedback with on-demand, validated patient education. c Virtual visits enabled by video, voice, and text features. 3. Clinician portals to allow for the care team to manage patient care including direct communication, tailoring medical plans, adjustments of device settings. 4. Connected wearable and implantable physiological sensors and devices. 5. Social connectivity to medical communities 
large organizations such as the NBA, though studies have yet to be completed and these solutions remain solely in the realm of the consumer [73, 74]. Recently, Masimo received FDA emergency clearance for a wireless, tetherless version of their critical care grade heart rate and pulse oximeter sensor (Radius PPG), as well as a continuous temperature patch (Radius T) that is crucially provided with a secure cloud-based consumer and provider-patient portal SafetyNet to enable RPM [75]. While the sensor technology is limited to a 7-day time period, the model of a patient-centric platform enabled by an automated alert system tailored specifically to the needs of the patient by the provider on a separate portal holds significant promise, and studies are under way to evaluate the ability of this program to aid in remote patient care.

\section{Conclusion}

The COVID-19 pandemic has ushered in a new era of cardiac management with RPM as its cornerstone finally brings the much-needed spark to ignite the digital disruption of medicine that has already revolutionized transportation, banking, and entertainment spheres. It is impossible to imagine a world where providers and patients will go back to the in-patient only model and the future will likely see a hybrid of inpatient care augmented by cloud-based, continuous home monitoring via implantable and/or wearable devices. Patientcentric platforms will provide patients access to their own real-time data, automated alerts, and personalized healthcare plans along with pertinent education empowering them to be participants in their own care. Conversely, healthcare teams will be provided data to identify new or worsening medical conditions allowing for earlier initiation or modification of treatment strategies with the goal of improving clinical outcomes and preventing the need for acute care (Fig. 1). Huge care gaps remain to realize the virtual, or lifecare, model of healthcare. Several barriers still stand in the way of virtual care model implementation including lack of infrastructure, inadequate reimbursement, uncertainty over future telehealth regulations, privacy concerns, and limited technical skills and lack of access due to poor internet access. Chronic disease management software, remote and continuous diagnostics, and digital COVID prevention and care digital tools are urgently needed. It is essential that the medical community guides this digital disruption by promoting validating technology and digital solutions using scientifically rigorous research standards and developing best practices to promote patient safety and well-being.

\section{Compliance with Ethical Standards}

Conflict of Interest The authors declare that they have no conflict of interest.
Human and Animal Rights and Informed Consent This article does not contain any studies with human or animal subjects performed by any of the authors.

\section{References}

Papers of particular interest, published recently, have been highlighted as:

- Of importance

•- Of major importance

1. Beck H, Boden WE, Patibandla S, Kireyev D, Gupta V, Campagna $\mathrm{F}$, et al. 50th anniversary of the first successful permanent pacemaker implantation in the United States: historical review and future directions. Am J Cardiol. 2010;106:810-8.

2. Saxon LA, Hayes DL, Gilliam FR, Heidenreich PA, Day J, Seth M, et al. Long-term outcome after ICD and CRT implantation and influence of remote device follow-up: the ALTITUDE survival study. Circulation. 2010;122:2359-67.

3. Akar JG, Bao H, Jones PW, Wang Y, Varosy PD, Masoudi FA, et al. Use of remote monitoring is associated with lower risk of adverse outcomes among patients with implanted cardiac defibrillators. Circ Arrhythmia Electrophysiol. 2015;8:1173-80.

4. Varma N, Piccini JP, Snell J, Fischer A, Dalal N, Mittal S. The relationship between level of adherence to automatic wireless remote monitoring and survival in pacemaker and defibrillator patients. $\mathrm{J}$ Am Coll Cardiol. 2015;65:2601-10.

5. Slotwiner D, Varma N, Akar JG, Annas G, Beardsall M, Fogel RI, et al. HRS expert consensus statement on remote interrogation and monitoring for cardiovascular implantable electronic devices. Hear Rhythm. 2015;12:e69-e100.

6.• Miller J, Bose R, Saxon LA. Chapter 63: Personal cardiac monitoring. In: Jalife J, Stevenson W, editors. Zipes \& Jalife cardiac electrophysiology: from cell to bedside, 8th edition. Elsevier Health Sciences; 2020. [In Print]

7. Centers for Medicare \& Medicaid Services. CMS payment policies $\&$ regulatory flexibilities during COVID-19 emergency. American Medical Association; 2020. https://www.ama-assn.org/practicemanagement/medicare/cms-payment-policies-regulatoryflexibilities-during-covid-19. Accessed 15 Aug 2020.

8. Lakkireddy DR, Chung MK, Gopinathannair R, Patton KK, Gluckman TJ, Turagam M, et al. Guidance for cardiac electrophysiology during the COVID-19 pandemic from the heart rhythm society COVID-19 task force; Electrophysiology section of the American College of Cardiology; and the Electrocardiography and Arrhythmias Committee of the Council on Clinical Cardiology, American Heart Association. Circulation. 2020;141(21):e823-31.

9. Abraham WT, Fiuzat M, Psotka MA, O'Connor CM. Heart failure collaboratory statement on heart failure remote monitoring in the landscape of COVID-19 and social distancing. JACC Hear Fail. 2020;8:692. This document provides recommendations pertaining to heart failure management and remote monitoring for patients during the COVD-19 pandemic.

10. Huynh K. Reduced hospital admissions for ACS - more collateral damage from COVID-19. Nat Rev Cardiol. 2020;17:453.

11. Yancy CW, Fonarow GC. Coronavirus disease 2019 (COVID-19) and the heart - is heart failure the next chapter? JAMA Cardiol. 2020. https://doi.org/10.1001/jamacardio.2020.3575.

12. Yee R, Verma A, Beardsall M, Fraser J, Philippon F, Exner DV. Canadian Cardiovascular Society/Canadian Heart Rhythm Society joint position statement on the use of remote monitoring for 
cardiovascular implantable electronic device follow-up. Can J Cardiol. 2013;29:644-51.

13.• Steinberg JS, Varma N, Cygankiewicz I, et al. 2017 ISHNE-HRS expert consensus statement on ambulatory ECG and external cardiac monitoring/telemetry. Ann Noninvasive Electrocardiol. 2017. https://doi.org/10.1111/anec.12447. This document developed by experts from the International Society for Holter and Noninvasive Electrocardiology and Heart Rhythm Society summarizes the background, provides the framework, and details the guidelines for how best to utilize amblatory electrocardiography techniques in clinical care.

14. Wilkoff BL, Auricchio A, Brugada J, Cowie M, Ellenbogen KA, Gillis AM, et al. HRS/EHRA expert consensus on the monitoring of cardiovascular implantable electronic devices (CIEDs): description of techniques, indications, personnel, frequency and ethical considerations. Europace. 2008;10:707-25.

15. Versteeg H, Timmermans I, Widdershoven J, Kimman GJ, Prevot $\mathrm{S}$, Rauwolf T, et al. Effect of remote monitoring on patient-reported outcomes in European heart failure patients with an implantable cardioverter-defibrillator: primary results of the REMOTE-CIED randomized trial. Europace. 2019;21:1360-8.

16. Hindricks G, Taborsky M, Glikson M, Heinrich U, Schumacher B, Katz A, et al. Implant-based multiparameter telemonitoring of patients with heart failure (IN-TIME): a randomised controlled trial. Lancet. 2014;384:583-90.

17. Petersen HH, Larsen MCJ, Nielsen OW, Kensing F, Svendsen JH. Patient satisfaction and suggestions for improvement of remote ICD monitoring. J Interv Card Electrophysiol. 2012;34:317-24.

18. Mabo P, Victor F, Bazin P, Ahres S, Babuty D, Da Costa A, et al. A randomized trial of long-term remote monitoring of pacemaker recipients (the COMPAS trial). Eur Heart J. 2012;33:1105-11.

19. Crossley GH, Chen J, Choucair W, Cohen TJ, Gohn DC, Johnson WB, et al. Clinical benefits of remote versus transtelephonic monitoring of implanted pacemakers. J Am Coll Cardiol. 2009;54: 2012-9.

20. Crossley GH, Boyle A, Vitense H, Chang Y, Mead RH. The CONNECT (clinical evaluation of remote notification to reduce time to clinical decision) trial: the value of wireless remote monitoring with automatic clinician alerts. J Am Coll Cardiol. 2011;57: 1181-9.

21. Varma N, Epstein AE, Irimpen A, Schweikert R, Love C. Efficacy and safety of automatic remote monitoring for implantable cardioverter-defibrillator follow-up: the lumos-t safely reduces routine office device follow-up (TRUST) trial. Circulation. 2010;122: 325-32.

22. Powell BD, Saxon LA, Boehmer JP, Day JD, Gilliam FR, Heidenreich PA, et al. Survival after shock therapy in implantable cardioverter-defibrillator and cardiac resynchronization therapydefibrillator recipients according to rhythm shocked: the altitude survival by rhythm study. J Am Coll Cardiol. 2013;62:1674-9.

23. Hayes DL, Boehmer JP, Day JD, Gilliam F III, Heidenreich PA, Seth M, et al. Cardiac resynchronization therapy and the relationship of percent biventricular pacing to symptoms and survival. Hear Rhythm. 2011;8:1469-75.

24. Morgan JM, Kitt S, Gill J, McComb JM, Ng GA, Raftery J, et al. Remotemanagement of heart failure using implantable electronic devices. Eur Heart J. 2017;38:2352-60.

25. Kurek A, Tajstra M, Gadula-Gacek E, et al. Impact of remote monitoring on long-term prognosis in heart failure patients in a realworld cohort: results from all-comers COMMIT-HF trial. J Cardiovasc Electrophysiol. 2017;28:425-31.

26. Parthiban N, Esterman A, Mahajan R, Twomey DJ, Pathak RK, Lau DH, et al. Remote monitoring of implantable cardioverter-defibrillators: a systematic review and meta-analysis of clinical outcomes. J Am Coll Cardiol. 2015;65:2591-600.
27. Glotzer TV, Daoud EG, Wyse DG, Singer DE, Ezekowitz MD, Hilker C, et al. The relationship between daily atrial tachyarrhythmia burden from implantable device diagnostics and stroke risk the trends study. Circ Arrhythmia Electrophysiol. 2009;2:474-80.

28. Healey JS, Connolly SJ, Gold MR, Israel CW, van Gelder I, Capucci A, et al. Subclinical atrial fibrillation and the risk of stroke. N Engl J Med. 2012;366:120-9.

29. Pietro RR, Vaccari D, Morichelli L, et al. Stroke incidence in patients with cardiac implantable electronic devices remotely controlled with automatic alerts of atrial fibrillation. A sub-analysis of the HomeGuide study. Int J Cardiol. 2016;219:251-6.

30. Martin DT, Bersohn MM, Waldo AL, Wathen MS, Choucair WK, Lip GYH, et al. Randomized trial of atrial arrhythmia monitoring to guide anticoagulation in patients with implanted defibrillator and cardiac resynchronization devices. Eur Heart J. 2015;36:1660-8.

31. Raj LM, Saxon LA. Haemodynamic monitoring devices in heart failure: maximising benefit with digitally enabled patient centric care. Arrhythmia Electrophysiol Rev. 2018;7:294-8.

32. FDA. Premarket Approval (PMA). 2014. https://www.accessdata. fda.gov/scripts/cdrh/cfdocs/cfpma/pma.cfm?id=P100045. Accessed 10 Mar 2020.

33. Adamson PB, Abraham WT, Bourge RC, Costanzo MR, Hasan A, Yadav $\mathrm{C}$, et al. Wireless pulmonary artery pressure monitoring guides management to reduce decompensation in heart failure with preserved ejection fraction. Circ Hear Fail. 2014;7:935-44.

34. Abraham WT, Adamson PB, Bourge RC, Aaron MF, Costanzo MR, Stevenson LW, et al. Wireless pulmonary artery haemodynamic monitoring in chronic heart failure: a randomised controlled trial. Lancet. 2011;377:658-66.

35. Abraham WT, Stevenson LW, Bourge RC, Lindenfeld JA, Bauman JG, Adamson PB. Sustained efficacy of pulmonary artery pressure to guide adjustment of chronic heart failure therapy: complete follow-up results from the CHAMPION randomised trial. Lancet. 2016;387:453-61.

36. Adamson PB, Ginn G, Anker SD, Bourge RC, Abraham WT. Remote haemodynamic-guided care for patients with chronic heart failure: a meta-analysis of completed trials. Eur J Heart Fail. 2017;19:426-33. This meta-analysis evaluates data from completed clinical trials evaluating the role of remote hemodynamic monitoring in patients with heart failure and concludes the superior treatment of "permanently implanted sensors and frequent filling pressure evaluation" in reducing the risk of longterm hospitalization for heart failure in comparison to traditional clinical management.

37. Desai AS, Bhimaraj A, Bharmi R, Jermyn R, Bhatt K, Shavelle D, et al. Ambulatory hemodynamic monitoring reduces heart failure hospitalizations in "real-world" clinical practice. J Am Coll Cardiol. 2017;69:2357-65.

38. Raval NY, Shavelle D, Bourge RC, Costanzo MR, Shlofmitz R, Heywood JT, et al. Significant reductions in heart failure hospitalizations with the pulmonary artery pressure guided HF system: preliminary observations from the CardioMEMS post approval study. J Card Fail. 2017;23:S27.

39. Shavelle DM, Desai AS, Abraham WT, et al. Lower rates of heart failure and all-cause hospitalizations during pulmonary artery pressure-guided therapy for ambulatory heart failure. Circ Hear Fail. 2020. https://doi.org/10.1161/circheartfailure.119.006863. This multi-center, prospective study is the largest of its kind to evaluate the outcomes of ambulatory hemodynamics moitoring of patients with implantable pulmonary artery sensors.

40. Sanna T, Diener H-C, Passman RS, di Lazzaro V, Bernstein RA, Morillo CA, et al. Cryptogenic stroke and underlying atrial fibrillation. N Engl J Med. 2014;370:2478-86.

41. Steinhubl SR, Waalen J, Edwards AM, Ariniello LM, Mehta RR, Ebner GS, et al. Effect of a home-based wearable continuous ECG 
monitoring patch on detection of undiagnosed atrial fibrillation the mSToPS randomized clinical trial. JAMA - J Am Med Assoc. 2018;320:146-55.

42. Steinhubl SR, Mehta RR, Ebner GS, Ballesteros MM, Waalen J, Steinberg G, et al. Rationale and design of a home-based trial using wearable sensors to detect asymptomatic atrial fibrillation in a targeted population: the mHealth screening to prevent strokes (mSToPS) trial. American Heart Journal. 2016;175:77-85.

43. Halcox JPJ, Wareham K, Cardew A, Gilmore M, Barry JP, Phillips $\mathrm{C}$, et al. Assessment of remote heart rhythm sampling using the AliveCor heart monitor to screen for atrial fibrillation the REHEARSE-AF study. Circulation. 2017;136:1784-94.

44. Lau JK, Lowres N, Neubeck L, Brieger DB, Sy RW, Galloway CD, et al. IPhone ECG application for community screening to detect silent atrial fibrillation: a novel technology to prevent stroke. Int $\mathbf{J}$ Cardiol. 2013;165:193-4.

45. Lowres N, Neubeck L, Salkeld G, Krass I, McLachlan AJ, Redfern $\mathrm{J}$, et al. Feasibility and cost-effectiveness of stroke prevention through community screening for atrial fibrillation using iPhone ECG in pharmacies: the SEARCH-AF study. Thromb Haemost. 2014;111:1167-76.

46. Chan PH, Wong CK, Pun L, Wong YF, Wong MMY, Chu DWS, et al. Head-to-head comparison of the AliveCor heart monitor and microlife WatchBP office AFIB for atrial fibrillation screening in a primary care setting. Circulation. 2017;135:110-2.

47. Bumgarner JM, Lambert CT, Hussein AA, Cantillon DJ, Baranowski B, Wolski K, et al. Smartwatch algorithm for automated detection of atrial fibrillation. J Am Coll Cardiol. 2018;71: 2381-8.

48. Statement from FDA commissioner Scott Gottlieb, M.D., and center for devices and radiological health director Jeff Shuren, M.D., J.D., on agency efforts to work with tech industry to spur innovation in digital health | FDA. https://www.fda.gov/news-events/pressannouncements/statement-fda-commissioner-scott-gottlieb-mdand-center-devices-and-radiological-health-director. Accessed 20 Aug 2020.

49. Perez MV, Mahaffey KW, Hedlin H, et al. Large-scale assessment of a smartwatch to identify atrial fibrillation. N Engl J Med. 2019;381:1909-17. This peer-reviewed publication shares the results of the Apple Heart Study demonstrating that the Apple Watch AF algorithm can successfully detect and the feasibility of completing a siteless study based on data from consumer owned devices.

50. ECG app and irregular heart rhythm notification available today on Apple Watch - Apple. https:/www.apple.com/newsroom/2018/12/ ecg-app-and-irregular-heart-rhythm-notification-available-todayon-apple-watch/. Accessed 26 Feb 2020.

51. Iqbal S Apple ResearchKit, HealthKit and CareKit, Health App what does it all mean? https://www.biotaware.com/blog/appleresearchkit-healthkit-and-carekit-health-app-what-does-it-allmean/. Accessed 26 Feb 2020.

52. (2020) About the Heartline Study by Johnson \& Johnson and Apple. https://www.heartline.com/about. Accessed 28 Aug 2020.

53. Telehealth: A post-COVID-19 reality?| McKinsey. https://www. mckinsey.com/industries/healthcare-systems-and-services/ourinsights/telehealth-a-quarter-trillion-dollar-post-covid-19-reality\#. Accessed 24 Aug 2020.

54. Shi S, Qin M, Shen B, Cai Y, Liu T, Yang F, et al. Association of cardiac injury with mortality in hospitalized patients with COVID19 in Wuhan, China. JAMA Cardiol. 2020;5:802-10.

55. Guo T, Fan Y, Chen M, Wu X, Zhang L, He T, et al. Cardiovascular implications of fatal outcomes of patients with coronavirus disease 2019 (COVID-19). JAMA Cardiol. 2020;5:1.

56. Wang D, Hu B, Hu C, Zhu F, Liu X, Zhang J, et al. Clinical characteristics of 138 hospitalized patients with 2019 novel coronavirus-infected pneumonia in Wuhan, China. JAMA - J Am Med Assoc. 2020;323:1061-9.

57. Goyal P, Choi JJ, Pinheiro LC, Schenck EJ, Chen R, Jabri A, et al. Clinical characteristics of Covid-19 in New York City. N Engl J Med. 2020;382:2372-4.

58. Bhatla A, Mayer MM, Adusumalli S, Hyman MC, Oh E, Tierney A, et al. COVID-19 and cardiac arrhythmias. Hear Rhythm. 2020;17:1439-44. https://doi.org/10.1016/j.hrthm.2020.06.016.

59. Dherange P, Lang J, Qian P, Oberfeld B, Sauer WH, Koplan B, et al. Arrhythmias and COVID-19: a review. JACC Clin Electrophysiol. 2020;6:1193-204. https://doi.org/10.1016/j.jacep. 2020.08.002.

60. $\mathrm{Hu} \mathrm{H}, \mathrm{Ma} \mathrm{F}$, Wei X, Fang Y. Coronavirus fulminant myocarditis saved with glucocorticoid and human immunoglobulin. Eur Heart J. 2020. https://doi.org/10.1093/eurheartj/ehaa190.

61. Driggin E, Madhavan MV, Bikdeli B, Chuich T, Laracy J, BiondiZoccai G, et al. Cardiovascular considerations for patients, health care workers, and health systems during the COVID-19 pandemic. J Am Coll Cardiol. 2020;75:2352-71.

62. Puntmann VO, Carerj ML, Wieters I, et al. Outcomes of cardiovascular magnetic resonance imaging in patients recently recovered from coronavirus disease 2019 (COVID-19). JAMA Cardiol. 2020. https://doi.org/10.1001/jamacardio.2020.3557. This publication shared cohort study results showing the increased risk of lasting cardiac effects in recently recovering from COVID-19 infection as evidenced by signs of ongoing cardiac inflammation in patients seen on MRI.

63. Minguito-Carazo C, Echarte-Morales J, Benito-González T, et al. QT interval monitoring with handheld heart rhythm ECG device in COVID-19 patients. 2020. https://doi.org/10.21203/rs.3.rs-38108/v1.

64. Giudicessi JR, Noseworthy PA, Friedman PA, Ackerman MJ. Urgent guidance for navigating and circumventing the QTcprolonging and torsadogenic potential of possible pharmacotherapies for coronavirus disease 19 (COVID-19). Mayo Clin Proc. 2020;95:1213-21

65. Mercuro NJ, Yen CF, Shim DJ, Maher TR, McCoy CM, Zimetbaum PJ, et al. Risk of QT interval prolongation associated with use of hydroxychloroquine with or without concomitant azithromycin among hospitalized patients testing positive for coronavirus disease 2019 (COVID-19). JAMA Cardiol. 2020;5:103641. https://doi.org/10.1001/jamacardio.2020.1834.

66. Remote or wearable patient monitoring devices EUAs FDA. https://www.fda.gov/medical-devices/coronavirus-disease-2019covid-19-emergency-use-authorizations-medical-devices/remoteor-wearable-patient-monitoring-devices-euas?utm_campaign= 2020-06-18NewEUACOVID-19page \&utm_medium= email\&utm source=Eloqua. Accessed 15 Aug 2020.

67. COVID-19 in-home monitoring program launched - Washington University School of Medicine in St. Louis. https://medicine.wustl. edu/news/covid-19-in-home-monitoring-program-launched/. Accessed 21 Jul 2020.

68. Medina M, Babiuch C, Card M, Gavrilescu R, Zafirau W, Boose E, et al. Home monitoring for COVID-19: posted April 18, 2020. Cleve Clin J Med. 2020;87:1-4.

69. Mayo clinic teams with medically home to expand in-home hospital care | FierceHealthcare. https://www.fiercehealthcare.com/ hospitals/mayo-clinic-teams-medically-home-to-expand-homehospital-car. Accessed 24 Aug 2020.

70. Apple Watch users can monitor their body's response to COVID19, flu with Cardiogram app | Appleinsider. https://appleinsider. com/articles/20/03/19/apple-watch-users-can-monitor-their-bodysresponse-to-covid-19-flu-with-cardiogram-app. Accessed 22 Jul 2020.

71. Radin JM, Wineinger NE, Topol EJ, Steinhubl SR. Harnessing wearable device data to improve state-level real-time surveillance of influenza-like illness in the USA: a population-based study. 
Lancet Digit Heal. 2020;2:e85-93. This publication highlights the possibility of monitoring viral outbreaks across wide populations through the use of digital surveillance tools, such as activity trackers and other wearable and mobile devices.

72. Natarajan A, Su H-W, Heneghan C. Assessment of physiological signs associated with COVID-19 measured using wearable devices. https://doi.org/10.1101/2020.08.14.20175265.

73. Heart Rate \& Valuable WHOOP Data in COVID-19|Coronavirus | WHOOP. https://www.whoop.com/thelocker/whoop-datacoronavirus/. Accessed 21 Jul 2020.

74. Oura partners with the NBA to help players, coaches, and staff understand their health as the season resumes. https://ouraring. com/partners-with-the-nba. Accessed 24 Aug 2020.
75. Masimo and university hospitals jointly announce Masimo SafetyNet ${ }^{\mathrm{TM}}$, a new remote patient monitoring solution University Hospitals Newsroom. https://news.uhhospitals.org/ news-releases/masimo-and-university-hospitals-jointly-announcemasimo-safetynet-a-new-remote-patient-monitoring-solution.htm. Accessed 24 Aug 2020.

Publisher's Note Springer Nature remains neutral with regard to jurisdictional claims in published maps and institutional affiliations. 